\title{
In-Hospital Clinical Outcomes of Elderly Patients ( $\geq 80$ Years) Undergoing Percutaneous Coronary Intervention
}

Takashi Miura, MD; Yusuke Miyashita, MD; Hirohiko Motoki, MD; Kentaro Shimada, MD; Masanori Kobayashi, MD; Hiroyuki Nakajima, MD; Hikaru Kimura, MD; Hiroshi Akanuma, MD;

Eiichiro Mawatari, MD; Toshio Sato, MD; Shoji Hotta, MD; Yuichi Kamiyoshi, MD;

Takuya Maruyama, MD; Noboru Watanabe, MD; Takayuki Eisawa, MD; Shinichi Aso, MD;

Shinichiro Uchikawa, MD; Naoto Hashizume, MD; Noriyuki Sekimura, MD; Takehiro Morita, MD; Soichiro Ebisawa, MD; Atsushi Izawa, MD; Takeshi Tomita, MD; Jun Koyama, MD; Uichi Ikeda, MD

\begin{abstract}
Background: The clinical outcomes of elderly patients ( $\geq 80$ years old) undergoing percutaneous coronary intervention $(\mathrm{PCl})$ has not been well established, despite recent advances in both devices and techniques.
\end{abstract}

\begin{abstract}
Methods and Results: We recruited patients from the SHINANO Registry, a prospective, observational, multicenter, cohort study. From August 2012 to July 2013, a total of 1,923 consecutive patients with 2,250 elective/urgent PCls (2,105 admissions) (mean age, $71 \pm 11$ years; $\geq 80$ years, $23 \%$; men, $77 \%$ ) were enrolled. The primary endpoint was procedural success. The secondary endpoints were in-hospital death and in-hospital major adverse cardiovascular events (MACE). The procedural success rate was significantly lower (83.7\% vs. $89.1 \%, P=0.0001)$, and the rates of in-hospital mortality and MACE were significantly higher in elderly than in non-elderly patients $(3.6 \%$ vs. $1.5 \%$, $\mathrm{P}=0.005 ; 4.4 \%$ vs. $2.3 \%, \mathrm{P}=0.016$, respectively). For elective $\mathrm{PCl}$, the rates of procedural success and in-hospital MACE were similar between groups ( $90.3 \%$ vs. $91.3 \%, P=0.65,2.3 \%$ vs. $1.2 \%, P=0.2$, respectively). On multivariate analysis, being elderly was not an independent predictor of procedural failure (OR, 1.15; $\mathrm{Cl}, 0.81-1.61 ; \mathrm{P}=0.43)$.
\end{abstract}

Conclusions: In elderly patients, $\mathrm{PCl}$ is safe and feasible. The presence of comorbidities is a more important factor than age alone. (Circ $J$ 2014; 78: 1097-1103)

Key Words: Coronary artery disease; Elderly; Percutaneous coronary intervention

W orldwide, the general population is aging, and octogenarians are the fastest growing population. ${ }^{1}$ Japan is one of the countries with an old population. The Cabinet Office of the Government of Japan reported that Japanese life expectancy would increase from 79.4 years in men and 85.9 years in women in 2011 to 84.2 years in men and 90.9 years in women in $2060 .^{2}$ Older age is an indepen- dent predictor for arteriosclerosis and cardiovascular events. ${ }^{3}$ Elderly patients tend to have extensive comorbidities and complex coronary lesions, which can make both the patient and clinician hesitant about percutaneous coronary interventions (PCI), even though the TIME study suggested that in elderly patients, revascularization treatment is safe and more effective than the optimal medical treatment for symptoms. ${ }^{4}$ New devices

Received February 2, 2014; revised manuscript received March 5, 2014; accepted March 6, 2014; released online March 21, 2014 Time for primary review: 18 days

Department of Cardiovascular Medicine, Shinshu University School of Medicine, Matsumoto (T. Miura, Y.M., H.M., N.H., S.E., A.I., T.T., J.K., U.I.); Department of Cardiology, Nagano Red Cross Hospital, Nagano (K.S., H.N.); Department of Cardiology, Matsumoto Kyoritsu Hospital, Matsumoto (M.K.); Department of Cardiology, Saku Central Hospital, Saku (H.K., E.M.); Department of Cardiology, Iida Municipal Hospital, Iida (H.A.); Department of Cardiology, Shinonoi General Hospital, Nagano (T.S., T. Maruyama); Department of Cardiology, Ina Central Hospital, Ina (S.H.); Department of Cardiology, Nagano Municipal Hospital, Nagano (Y.K.); Department of Cardiology, Hokushin General Hospital, Nakano (N.W.); Department of Cardiology, Komoro Kosei General Hospital, Komoro (T.E.); Department of Cardiology, Aizawa Hospital, Matsumoto (S.A.); Department of Cardiology, Azumino Red Cross Hospital, Azumino (S.U.); Department of Cardiology, Okaya Municipal Hospital, Okaya (N.S.); and Department of Cardiology, Nagano Matsushiro General Hospital, Nagano (T. Morita), Japan

This paper was presented at the $78^{\text {th }}$ Annual Scientific Meeting of the Japanese Circulation Society, Late Breaking Cohort Studies 1-4 (March 21, 2014, Tokyo, Japan).

Mailing address: Takashi Miura, MD, Department of Cardiovascular Medicine, Shinshu University School of Medicine, 3-1-1 Asahi, Matsumoto 390-8621, Japan. E-mail: miuramen10miuramen@yahoo

ISSN-1346-9843 doi:10.1253/circj.CJ-14-0129

All rights are reserved to the Japanese Circulation Society. For permissions, please e-mail: cj@j-circ.or.jp 
and techniques for PCI have been developed in recent years, including a debulking device, drug-eluting stents (DES), a transradial approach, and sheathless guiding catheter for less invasive PCI. However, the clinical outcomes of elderly patients undergoing PCI has not yet been well established. It is important to update Japanese real-world data for the management of elderly patients undergoing PCI in the contemporary era.

Nagano Prefecture in Japan has the highest life expectancy among all prefectures. The Shinshu Prospective Multi-center Analysis for Elderly Patients with Coronary Artery Disease Undergoing Percutaneous Coronary Intervention (SHINANO) Registry was initiated to evaluate the clinical outcome, treatment, and clinical and lesion characteristics in elderly patients undergoing PCI.

This study presents the differences in baseline characteristics and short-term outcomes after initial PCI between elderly and non-elderly patients in the SHINANO Registry. We evaluated in-hospital clinical outcomes of elderly patients undergoing PCI.

\section{Methods}

\section{Study Design}

The SHINANO Registry is a prospective, multicenter, observational registry designed to provide up to 12 months of clinical follow-up. Between August 2012 and July 2013, a total of 1,923 consecutive patients with 2,250 PCIs (2,105 admissions) for any coronary artery disease (stable angina, ST-segment elevation myocardial infarction [STEMI], non-STEMI [NSTEMI], and unstable angina [UA]) were enrolled from 16 institutions in Nagano prefecture. This study had no exclusion criteria and was an all-comer registry.

Patients were enrolled after approval of each hospital's ethics committee and after informed consent was given by the patients. This study was performed in accordance with the Declaration of Helsinki. The SHINANO Registry is registered with the University Hospital Medical Information NetworkClinical Trials Registry (UMIN-CTR), as accepted by the International Committee of Medical Journal Editors (No. UMIN 000010070).

\section{Endpoints}

The primary endpoint was procedural success. The secondary endpoints were in-hospital death and in-hospital major adverse cardiovascular events (MACE; all-cause death, myocardial infarction $[\mathrm{MI}]$, stroke, and stent thrombosis).

\section{Study Procedure}

All patients were pretreated with ticlopidine (200 mg daily) or clopidogrel (75 mg daily), in addition to aspirin (100-200 mg daily). A loading dose of $300 \mathrm{mg}$ of clopidogrel was administered to clopidogrel-naïve patients. Aspirin and thienopyridines were started at least 2 days before elective stenting. The interventional strategy and stent selection were left to the discretion of the operator in all procedures.

\section{Definitions}

Elderly age was defined as $\geq 80$ years old. Acute coronary syndrome (ACS) is a composite of STEMI, NSTEMI and UA. STEMI was diagnosed in patients with chest symptoms, STsegment elevation $\geq 1 \mathrm{mV}$ in 2 or more limb leads or $\geq 2$ contiguous precordial leads or left bundle branch block, and elevated biochemical markers of myocardial necrosis (troponin $\mathrm{T}$ levels $\geq 0.1 \mathrm{ng} / \mathrm{ml}$ or creatine phosphokinase 2-fold above the normal range). NSTEMI was diagnosed in patients with chest symptoms, ST-segment depression $\leq 0.05 \mathrm{mV}$, T-wave inver- sion $\geq 0.3 \mathrm{mV}$, or transient $<0.05 \mathrm{mV}$ ST-segment elevation, and elevated biochemical markers of myocardial necrosis (and no ECG abnormalities for STEMI). UA was diagnosed in patients with persistent resting or nocturnal chest pain together with additional findings. Angiographic success was defined as achievement of a minimum stenosis diameter reduction to less than $20 \%$ with grade 3 Thrombolysis In Myocardial Infarction (TIMI) flow. Procedural success was defined as achievement of angiographic success without any complications during hospitalization. Procedural failure was defined as the inability to complete PCI or any complications during hospitalization. Complications were defined as all-cause mortality, procedure-related stroke, procedure-related MI, bleeding complications, cholesterol crystal embolization (CCE), worsened renal function, coronary artery perforation or rupture, coronary dissection, stent thrombosis, final TIMI flow $\leq 2$ from any coronary event, or any other event requiring prolonged hospitalization. Bleeding complications were defined as blood transfusion or prolonged hospitalization owing to hematoma, gastrointestinal bleeding, or intracranial bleeding. Procedure-related MI was defined as creatine kinase (CK) or CK-MB above the upper limit of normal at each hospital or as the development of significant $Q$ waves in at least 2 contiguous leads of the ECG. Coronary artery disease (CAD) was defined as $>50 \%$ stenosis in a coronary vessel on angiography, history of coronary artery bypass graft surgery or PCI, or previous MI. Stroke was defined as ischemic stroke that persisted for $\geq 24 \mathrm{~h}$ and was diagnosed by a neurologist. Heart failure (HF) was based on a previous diagnosis of HF, history of hospitalization for $\mathrm{HF}$, or current treatment for HF. Diabetes was defined as HbA1c $\geq 6.5 \%$, casual plasma glucose $\geq 200 \mathrm{mg} / \mathrm{dl}$ or treatment with oral hypoglycemic agents or insulin injection. Hypertension was defined as systolic blood pressure (BP) $\geq 140 \mathrm{mmHg}$, diastolic $\mathrm{BP} \geq 90 \mathrm{mmHg}$, or ongoing therapy for hypertension. Dyslipidemia was defined as a serum total cholesterol concentration $\geq 220 \mathrm{mg} / \mathrm{dl}$, a low-density lipoproteincholesterol concentration $\geq 140 \mathrm{mg} / \mathrm{dl}$, or current treatment with lipid-lowering therapy. Body mass index was defined as weight in kilograms divided by the square of height in meters. Chronic kidney disease was defined as creatinine clearance $<30 \mathrm{ml} / \mathrm{min}$ estimated using the Cockcroft-Gould formula. Left ventricular ejection fraction (LVEF) was measured by echocardiography and LVEF $\leq 40 \%$ indicated LV dysfunction. Multivessel disease was defined as the presence of a $\geq 75 \%$ lesion in $\geq 2$ major coronary arteries. Documented peripheral artery disease (PAD) consisted of 1 or both criteria: current intermittent claudication with an ankle-brachial index $\leq 0.9$ or a history of intermittent claudication with a previous and related intervention, such as peripheral arterial bypass graft, stenting, angioplasty, atherectomy, or other vascular interventions, including amputation.

\section{Statistical Analysis}

Continuous variables are reported as mean \pm standard deviation. Categorical variables are reported as frequencies and percentages. Patient characteristics of the 2 age groups were compared using chi-squared tests. Continuous variables were compared by t-test. For each age group, multivariate logistic proportional hazard models were used to adjust for the effects of baseline risk factors on initial PCI success. $\mathrm{P}<0.05$ was considered statistically significant in all analyses.

\section{Results}

\section{Baseline Demographics}

Patient characteristics are shown in Table 1 . Of the 1,923 patients, 441 (22.9\%) were elderly ( $\geq 80$ years old, 37 patients $\geq 90$ 


\begin{tabular}{|c|c|c|c|c|}
\hline & Overall & Age $\geq 80$ years & Age $<80$ years & $P$ value \\
\hline $\mathrm{n}$ & 1,923 & 441 & 1,482 & \\
\hline Mean age (years) & $70.7 \pm 11.0$ & $84.1 \pm 3.4$ & $66.6 \pm 9.1$ & $<0.0001$ \\
\hline Male (\%) & $1,471(76.5)$ & $257(58.3)$ & $1,214(81.9)$ & $<0.0001$ \\
\hline BMI & $23.8 \pm 3.6$ & $22.3 \pm 3.3$ & $24.2 \pm 3.6$ & $<0.0001$ \\
\hline Hypertension (\%) & 1,388 (72.2) & 348 (78.9) & $1,040(70.2)$ & $<0.0001$ \\
\hline Dyslipidemia (\%) & 1,147 (59.7) & $209(47.4)$ & $938(63.3)$ & $<0.0001$ \\
\hline Diabetes (\%) & $704(36.6)$ & $132(30.0)$ & $572(38.6)$ & 0.001 \\
\hline Oral (\%) & 441 (22.9) & $86(19.5)$ & $355(24.0)$ & 0.059 \\
\hline Insulin (\%) & $142(7.4)$ & $24(5.4)$ & $118(8.0)$ & 0.081 \\
\hline $\mathrm{Hb}$ & $13.9 \pm 5.9$ & $12.9 \pm 4.0$ & $14.4 \pm 6.8$ & $<0.0001$ \\
\hline eGFR & $61.5 \pm 24.4$ & $53.9 \pm 21.7$ & $63.5 \pm 23.8$ & $<0.0001$ \\
\hline Hemodialysis (\%) & $112(5.8)$ & $17(3.9)$ & $95(6.4)$ & 0.047 \\
\hline Current smoker (\%) & $354(18.4)$ & $30(6.8)$ & 324 (21.9) & $<0.0001$ \\
\hline Previous smoker (\%) & $974(50.7)$ & $158(35.8)$ & $816(55.1)$ & $<0.0001$ \\
\hline Previous MI (\%) & $485(25.2)$ & $114(25.9)$ & $371(25.0)$ & 0.69 \\
\hline Previous CABG (\%) & $146(7.6)$ & $30(6.8)$ & $116(7.8)$ & 0.49 \\
\hline Previous stroke (\%) & $183(9.5)$ & $64(14.5)$ & $119(8.0)$ & $<0.0001$ \\
\hline Previous intracranial bleeding (\%) & $28(1.5)$ & $13(3.0)$ & $15(1.0)$ & 0.003 \\
\hline PAD (\%) & $197(10.2)$ & $65(14.7)$ & $132(8.9)$ & $<0.0001$ \\
\hline Heart failure (\%) & $256(13.3)$ & $91(20.6)$ & $165(11.1)$ & $<0.0001$ \\
\hline $\mathrm{AF}(\%)$ & $208(10.8)$ & $76(17.2)$ & $132(8.9)$ & $<0.0001$ \\
\hline $\operatorname{LVEF}(\%)$ & $60.1 \pm 13.4$ & $58.8 \pm 13.8$ & $60.4 \pm 13.3$ & 0.041 \\
\hline LV dysfunctiona (\%) & $171(8.9)$ & $48(10.9)$ & $123(8.3)$ & 0.073 \\
\hline \multicolumn{5}{|l|}{ Medication at discharge } \\
\hline $\mathrm{n}$ & 2,063 & 460 & 1,603 & \\
\hline Aspirin (\%) & $1,962(95.1)$ & $431(93.7)$ & $1,531(95.5)$ & 0.88 \\
\hline Thienopyridines (\%) & $1,823(88.4)$ & $399(86.7)$ & $1,424(88.8)$ & 0.73 \\
\hline EPA (\%) & $79(3.8)$ & $10(2.1)$ & $69(4.3)$ & 0.04 \\
\hline Statins (\%) & $1,464(71.0)$ & $284(61.7)$ & $1,180(73.6)$ & $<0.0001$ \\
\hline ACEI/ARB (\%) & $1,350(65.4)$ & $287(62.4)$ & $1,063(66.3)$ & 0.23 \\
\hline$\beta$-blocker (\%) & $841(40.8)$ & $164(35.7)$ & 677 (42.2) & 0.017 \\
\hline Warfarin (\%) & $219(10.6)$ & $54(11.7)$ & $165(10.3)$ & 0.32 \\
\hline NOAC (\%) & $32(1.6)$ & $5(1.1)$ & $27(1.7)$ & 0.38 \\
\hline
\end{tabular}

aLV dysfunction was defined as $\leq 40 \%$ of LVEF.

$\mathrm{ACEl}$, angiotensin-converting enzyme inhibitor; AF, atrial fibrillation; ARB, angiotensin receptor blocker; BMI, body mass index; CABG, coronary artery bypass grafting; EPA, eicosapentaenoic acid; LVEF, left ventricular ejection fraction; MI, myocardial infarction; NOAC, new oral anticoagulant drug; PAD, peripheral arterial disease.

years old) and 1,482 (77.1\%) were non-elderly. Mean age was $84.1 \pm 3.4$ years in the elderly group and $66.6 \pm 9.1$ years in the non-elderly group. The elderly group had a significantly lower percentage of men $(58.3 \%$ vs. $81.9 \%, \mathrm{P}<0.0001)$. In terms of comorbidities, dyslipidemia, diabetes, hemodialysis, current smoking, and previous smoking were observed more frequently in the non-elderly patients. On the other hand, the elderly patients had higher rates of hypertension, previous stroke, previous cranial bleeding, PAD, previous HF, and arterial fibrillation than the non-elderly patients. LVEF was not significantly different between the elderly and non-elderly groups (59.1 $\pm 13.4 \%$ vs. $60.5 \pm 13.4 \%, \mathrm{P}=0.091)$. The elderly patients presented with ACS more frequently than the non-elderly patients $(44.2 \%$ vs. $39.6 \%, \mathrm{P}=0.046)$.

\section{Angiographic Findings}

The characteristics of the lesions and procedures are shown in Table 2. Similar lesional characteristics, including multivessel disease, left main trunk disease, bifurcation lesions, and chronic total occlusions, were found in both groups. However, the percentage of calcified lesions and ostial lesions was significantly higher in the elderly group. As a result, the SYNTAX score was significantly higher in the elderly group than in the non-elderly group (13.5 \pm 9.0 vs. $12.5 \pm 8.7, \mathrm{P}=0.032)$.

\section{In-Hospital Complications}

There were 220 complications during hospitalizations; 157 cases of complications during the PCI procedure $(6.9 \%)$, including coronary perforation in 23 patients $(1.0 \%)$, PCI-related stroke in 2 patients $(0.08 \%)$, and final TIMI flow $\leq 2$ caused by distal embolization in 139 patients $(6.2 \%)$. The rate of total complications during PCI was not significantly different between the elderly and non-elderly patients $(8.1 \%$ vs. $6.6 \%, \mathrm{P}=0.24)$. In terms of each complication during PCI, the incidences of coronary perforation, PCI-related stroke, and final TIMI flow $\leq 2$ were similar between groups $(1.0 \%$ vs. $1.0 \%, \mathrm{P}=0.89,0 \%$ vs. $0.1 \%, \mathrm{P}=0.44,7.6 \%$ vs. $5.8 \%, \mathrm{P}=0.14$, respectively). Furthermore, the angiographic success rate was not significantly different between groups ( $90.7 \%$ vs. $92.9 \%, \mathrm{P}=0.098)$, despite the difference in lesion complexity between the elderly and non- 


\begin{tabular}{|lcccc|}
\hline Table 2. Lesional Characteristics & & & & \\
$\mathrm{n}$ & Overall & Age $\geq \mathbf{8 0}$ years & Age $<80$ years & P value \\
ACS & 2,250 & 516 & 1,734 & \\
Multivessel disease (\%) & $914(40.6)$ & $228(44.2)$ & $686(39.6)$ & 0.046 \\
De novo & $928(41.2)$ & $220(42.6)$ & $709(40.9)$ & 0.479 \\
DES ISR & $1,971(87.6)$ & $454(88.5)$ & $1,517(87.6)$ & 0.579 \\
BMS ISR & $108(4.8)$ & $21(4.1)$ & $87(5.0)$ & 0.377 \\
LMT lesions & $165(7.3)$ & $41(8.0)$ & $124(7.2)$ & 0.549 \\
Calcified lesions & $63(2.8)$ & $17(3.3)$ & $46(2.7)$ & 0.438 \\
Ostial lesions & $658(29.3)$ & $180(35.2)$ & $478(27.8)$ & 0.001 \\
Bifurcation lesions & $174(7.7)$ & $55(10.7)$ & $119(6.9)$ & 0.005 \\
CTO lesions & $602(26.8)$ & $131(25.5)$ & $471(27.4)$ & 0.396 \\
SYNTAX score & $174(7.7)$ & $39(7.6)$ & $135(7.8)$ & 0.865 \\
PCI procedure & $12.7 \pm 8.8$ & $13.5 \pm 9.0$ & $12.5 \pm 8.7$ & 0.032 \\
TFI (\%) & & & & 0.35 \\
TBI (\%) & $680(30.2)$ & $162(31.4)$ & $518(29.9)$ & $68(3.9)$ \\
TRI (\%) & $87(3.9)$ & $19(3.7)$ & $1,099(63.4)$ & 0.29 \\
BMS use (\%) & $1,423(63.2)$ & $324(62.8)$ & $602(34.7)$ & 0.23 \\
DES use (\%) & $796(35.4)$ & $194(37.6)$ & $842(48.6)$ & 0.612 \\
\hline
\end{tabular}

ACS, acute coronary syndrome; BMS, bare metal stent; CTO, chronic total occlusion; DES, drug-eluting stent; ISR, in-stent restenosis; LMT, left main trunk; TBI, transbrachial intervention; TFI, transfemoral intervention, TRI, transradial intervention.

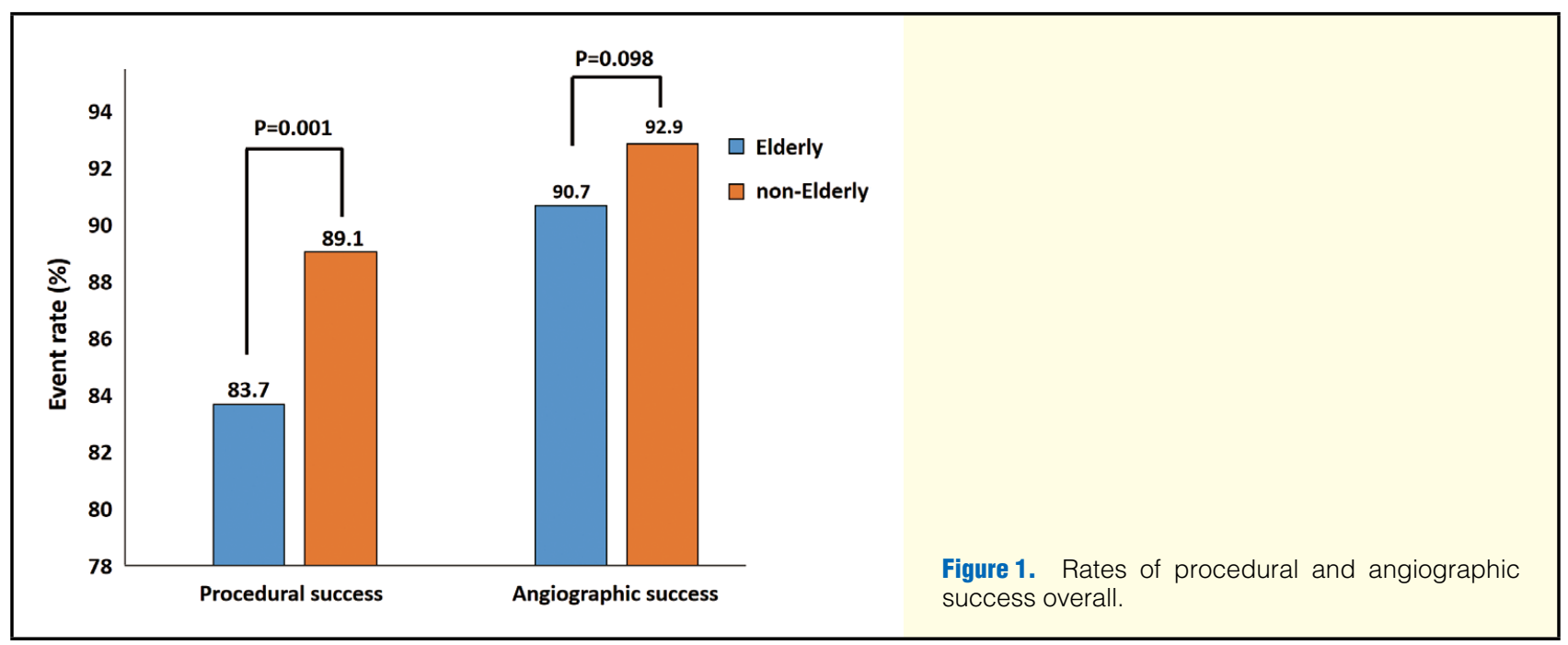

elderly patients.

\section{In-Hospital Procedural Success}

The angiographic success rate and the rate of complications during PCI were not significantly different between the elderly and non-elderly groups. However, the total complications during hospitalization were significantly higher in the elderly group than in the non-elderly group $(15.1 \%$ vs. $9.8 \%, \mathrm{P}=0.001)$. Therefore, the procedural success rate was significantly lower in the elderly group than in the non-elderly group (Figure 1). In patients with ACS, the procedural and angiographic success rates were significantly lower in the elderly group than in the nonelderly group $(75.8 \%$ vs. $85.8 \%, \mathrm{P}=0.001,85.1 \%$ vs. $90.4 \%$, $\mathrm{P}=0.008$ ) (Figure 2A).

Among the patients with non-ACS, the procedural and angiographic success rates were not significantly different between the elderly and non-elderly groups $(90.3 \%$ vs. $91.3 \%$,
$\mathrm{P}=0.61,95.1 \%$ vs. $94.6 \%, \mathrm{P}=0.70$ ) (Figure $2 \mathrm{~B}$ ).

Regarding in-hospital deaths, there were 42 cases during hospitalization, including cardiac death in 31 patients $(1.5 \%$; 29 presented with ACS, 2 presented with non-ACS), and, noncardiac death in 11 patients $(0.5 \%)$. Non-cardiac deaths included gastrointestinal bleeding ( 3 patients, $0.14 \%$ ), intracranial bleeding ( 2 patients, $0.1 \%$ ), sepsis ( 2 patients, $0.1 \%$ ), ischemic colitis ( 1 patients, $0.05 \%)$, hematologic malignancy ( 1 patients, $0.05 \%$ ), and pneumonia ( 2 patients, $0.1 \%$ ). The in-hospital mortality rate was significantly higher in the elderly group than in the non-elderly group (Figure 3). Regarding the in-hospital MACE, the incidence of PCI-related MI, PCI-related stroke, and stent thrombosis was not significantly different between the 2 groups $(0.4 \%$ vs. $0.4 \%, \mathrm{P}=0.87,0.4 \%$ vs. $0.4 \%, \mathrm{P}=0.87,0.6 \%$ vs. $0.2 \%, \mathrm{P}=0.11$, respectively). However, the rate of in-hospital MACE was significantly higher in the elderly group than in the non-elderly group (Figure 3). Furthermore, the duration 
A

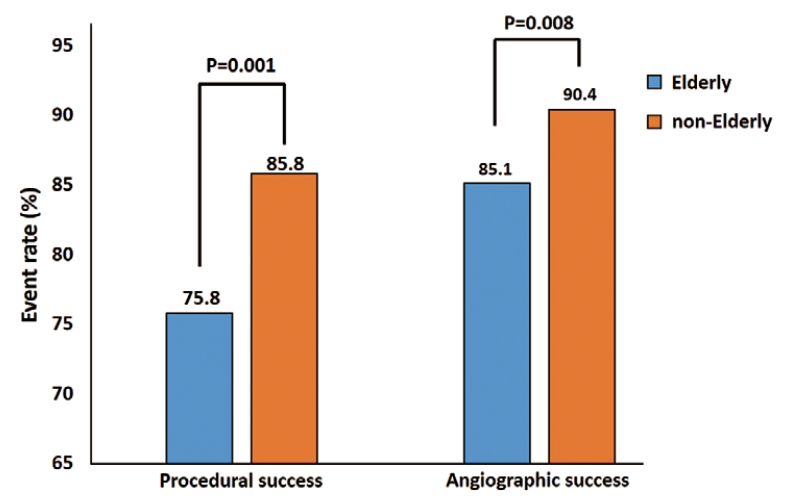

B

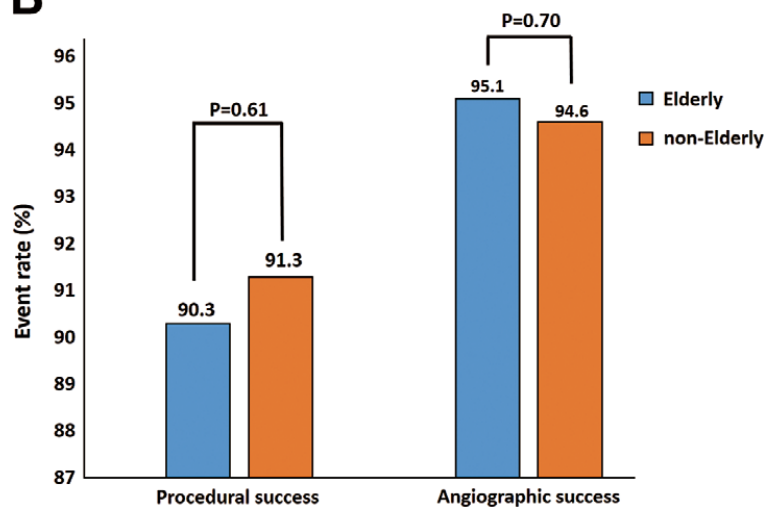

Figure 2. Rates of procedural success and angiographic success in ACS group (A) and non-ACS group (B). ACS, acute coronary syndrome.

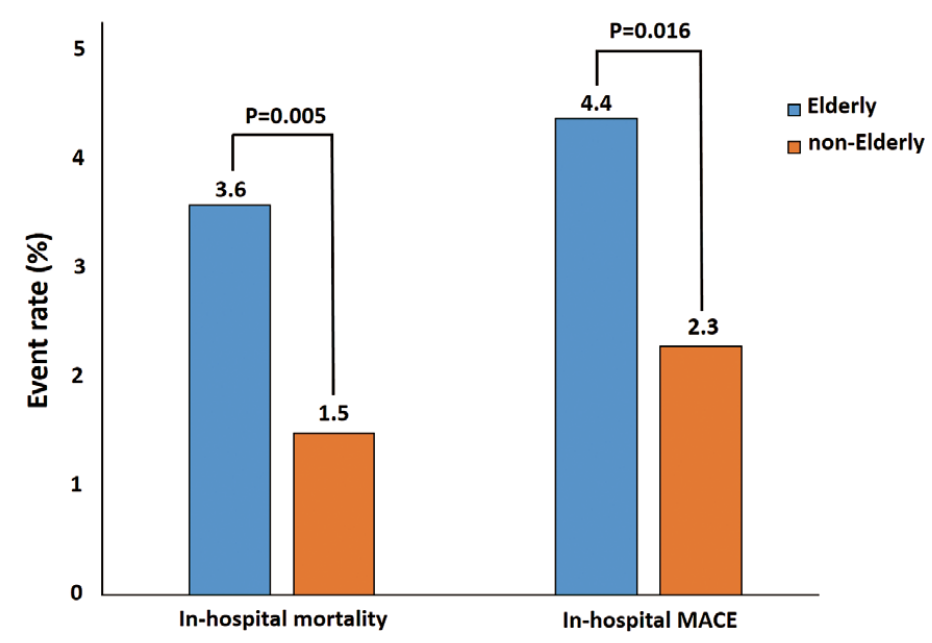

Figure 3. Rates of in-hospital mortality and in-hospital major adverse cardiovascular events (MACE) overall.

of hospitalization was significantly longer in the elderly group than in the non-elderly group $(15.9 \pm 23.7$ days vs. $10.7 \pm 16.0$ days, $\mathrm{P}<0.0001)$.

\section{Predictors of Procedural Failure and MACE}

Logistic regression analysis was performed to evaluate the power of age and other variables on procedural failure (Table 3 ) and MACE (Table 4). On multivariate analysis, older age was not an independent predictor of procedural failure and MACE. The presence of LV dysfunction, previous intracranial bleeding, and atrial fibrillation, ACS at admission, and estimated glomerular filtration rate were independent predictors of procedural failure and MACE. The SYNTAX score was an independent predictor of procedural failure, but had only a tendency to be an independent predictor of MACE.

\section{Discussion}

The SHINANO Registry is a large-scale, prospective, multicenter, observational registry of all CAD patients who have undergone PCI at regional cardiac centers, not including highvolume PCI centers. Each institution had 100-500 PCI cases/ year. Therefore, this registry revealed early outcomes of contemporary application of PCI in elderly patients treated in Japanese standard city hospitals. Approximately $25 \%$ of the patients were $\geq 80$ years old in our study. There were more women at an advanced age, as in previous reports. ${ }^{5}$

\section{Baseline Characteristics and Angiographic Findings}

The elderly patients had more severe coronary lesions and more extensive comorbidities than the non-elderly patients in this study. The prevalence of diabetes, smoking, and hyperlipidemia was significantly lower in the elderly group than in the nonelderly group, which is consistent with previous studies of elderly patients. ${ }^{6-8}$ This result suggests that patients with severe diabetes, hyperlipidemia, and who smoke tend to die earlier from their comorbidities.

\section{In-Hospital Adverse Outcomes and Complications}

The angiographic success rate was similar in both groups; however, the elderly group had a lower procedural success rate, a longer duration of hospitalization, and a higher rate of in-hospital mortality and ischemic events than the non-elderly group.

Previous reports show that elderly patients who undergo PCI 


\begin{tabular}{llccc|}
\hline \multicolumn{2}{l}{ Table 3. Univariate and Multivariate Predictors of Procedural Failure } & & \\
Variable & Unadjusted OR (95\% Cl) & P value & Adjusted OR (95\% Cl) & P value \\
$\quad$ Elderly & $1.59(1.19-2.13)$ & 0.002 & $1.15(0.81-1.61)$ & 0.43 \\
Female & $1.48(1.11-1.97)$ & 0.008 & $1.28(0.91-1.79)$ & 0.15 \\
BMI <18 & $1.50(0.81-2.75)$ & 0.20 & & \\
eGFR & $0.99(0.98-0.99)$ & $<0.0001$ & $0.99(0.98-0.99)$ & 0.005 \\
Multivessel disease & $1.35(1.504-1.75)$ & 0.026 & & \\
LV dysfunctiona & $2.63(1.82-3.79)$ & $<0.0001$ & $1.78(1.16-2.74)$ & 0.009 \\
Previous heart failure & $1.64(1.17-2.30)$ & 0.004 & & \\
ACS & $2.07(1.59-2.70)$ & $<0.0001$ & $1.99(1.48-2.70)$ & $<0.0001$ \\
Previous intracranial bleeding & $3.95(1.81-8.58)$ & 0.001 & $3.62(1.53-8.53)$ & 0.003 \\
AF & $1.85(1.28-2.66)$ & 0.001 & $1.87(1.25-2.80)$ & 0.003 \\
SYNTAX Score & $1.05(1.04-1.07)$ & $<0.0001$ & $1.04(1.03-1.04)$ & $<0.0001$ \\
Calcified lesions & $1.52(1.16-2.00)$ & 0.003 & & \\
Ostial lesions & $1.70(1.11-2.61)$ & 0.014 & & \\
LMT lesions & $2.61(1.43-4.78)$ & 0.002 & & \\
\hline
\end{tabular}

Elderly was defined as $\geq 80$ years old. aLV dysfunction was defined as $\geq 40 \%$ of LVEF.

$\mathrm{Cl}$, confidence interval; eGFR, estimated glomerular filtration rate; OR, odds ratio. Other abbreviations as in Tables 1,2.

\begin{tabular}{|lcccc|}
\hline \multicolumn{2}{l}{ Table 4. Univariate and Multivariate Predictors of In-Hospital Major Adverse Cardiac Events } & \\
Variable & Unadjusted OR (95\% Cl) & P value & Adjusted OR (95\% Cl) & P value \\
Elderly & $1.93(1.12-3.32)$ & 0.018 & $1.09(0.56-2.13)$ & 0.80 \\
eGFR & $0.98(0.97-0.99)$ & 0.001 & $0.99(0.97-0.99)$ & 0.03 \\
Multivessel disease & $1.80(1.07-3.03)$ & 0.027 & & \\
LV dysfunctiona & $6.42(3.61-11.40)$ & $<0.0001$ & $5.33(2.75-10.35)$ & $<0.0001$ \\
Previous heart failure & $2.84(1.61-5.02)$ & $<0.0001$ & & 0.001 \\
Previous intracranial bleeding & $5.87(1.98-17.44)$ & 0.001 & $7.22(2.18-23.89)$ & \\
AF & $2.24(1.17-4.29)$ & 0.015 & & 0.002 \\
ACS & $3.48(1.96-6.16)$ & $<0.0001$ & $2.96(1.50-5.81)$ & \\
TIMI $\leq 2$ & $5.27(2.89-9.60)$ & $<0.0001$ & & 0.059 \\
SYNTAX score & $1.047(1.022-1.072)$ & $<0.0001$ & $1.027(0.99-1.06)$ & \\
Ostial lesions & $2.34(1.23-4.85)$ & 0.023 & & \\
LMT lesions & $5.27(2.28-12.16)$ & $<0.0001$ & & \\
\hline
\end{tabular}

Elderly was defined as $\geq 80$ years old. aLV dysfunction was defined as $\geq 40 \%$ of LVEF.

TIMI, Thrombolysis In Myocardial Infarction. Other abbreviations as in Tables 1,2,3.

have a higher rate of in-hospital mortality, ischemic events, and complications and longer hospitalization than non-elderly patients. ${ }^{6-10}$ Appleby et al $^{11}$ reported that in-hospital mortality was significantly higher in very elderly patients ( $\geq 85$ years old) than in patients $<85$ years old $(6.93 \%$ vs. $1.20 \%, \mathrm{P}<0.0001)$. Renal, neurological, and bleeding complications were all greater in the very elderly cohort. In the current study, in-hospital mortality was significantly higher in the elderly patients than in the non-elderly patients who underwent PCI. Furthermore, very elderly patients ( $\geq 85$ years old) had higher mortality $(5 \%$ vs. $1.7 \%, \mathrm{P}=0.003$ ). However, renal, neurological, and bleeding complications were similar between the elderly and nonelderly groups in the present study. Similar results were observed for very elderly patients and not-very elderly patients ( $<85$ years old $)(2.3 \%$ vs. $1.3 \%, \mathrm{P}=0.21 ; 0 \%$ vs. $0.1 \% ; \mathrm{P}=0.66$, $2.3 \%$ vs. $1.6 \% ; \mathrm{P}=0.72$ ). When comparing our results with those of other studies, our results for in-hospital adverse outcomes and PCI-related complications in the elderly and very elderly patients appear to be acceptable.

\section{Predictors of Procedural Failure and MACE}

Our study revealed that older age is not a strong predictor of procedural failure and in-hospital ischemic events, despite the 2 -fold increase in both the procedural failure rate and the MACE rate in the elderly group compared with the non-elderly group. The elderly patients had more extensive comorbidities (renal insufficiency, PAD, previous HF, atrial fibrillation, and previous stroke and intracranial bleeding) and clinical presentation (ACS). These factors were stronger predictors of procedural failure and in-hospital ischemic events than age, which is consistent with previous studies of elderly patients. ${ }^{7,12,13}$ In particular, the rates of procedural failure and MACE in the patients with non-ACS were acceptable in both groups. Thus, the decision to perform PCI in elderly patients should not be based on age alone. The presence of comorbidities should also be considered.

\section{PCI Strategy in Elderly Patients}

DES are more suitable than bare meal stents (BMS) for the treatment of elderly patients with more complex coronary disease. 
In the current study, the SYNTAX score was significantly higher in the elderly patients than in the non-elderly patients. The Xima trial revealed that the incidence of MI and target vessel revascularization was lower (without an increased incidence of major hemorrhage) with the use of second-generation DES than with BMS. ${ }^{14}$ In that trial, the patients with DES were prescribed dual antiplatelet therapy (DAPT) for 1 year. Recently, it was shown that in patients with stable CAD or low-risk ACS treated with second-generation DES, 3 months of DAPT was noninferior to 12 months of DAPT for MACE or major bleeding, without significantly increasing the risk of stent thrombosis. ${ }^{15}$ Therefore, the length of DAPT for DES has become shorter worldwide. Accordingly, elderly patients with CAD may be treated with DES, if they can be treated with short-term DAPT. In the current study, the in-hospital bleeding complication rate was similar between the elderly and non-elderly patients. That result suggests that short-term DAPT in elderly patients with DES is safe, in terms of bleeding complications. In the current study, the percentage of DES was similar between the elderly and non-elderly patients, and was also similar between the 2 groups of elective PCI $(60.4 \%$ vs. $59.6 \%, \mathrm{P}=0.81)$ and urgent PCI $(30.7 \%$ vs. $31.6 \%, \mathrm{P}=0.79)$.

Prior reports have consistently documented that the transradial approach in primary PCI has the strong, important benefit of reducing vascular complications as compared with the transfemoral approach in STEMI patients. ${ }^{16}$ In contrast, elderly patients tend to have more severe arteriosclerosis. Our study found that the prevalence of PAD and the rate of previous stroke were significantly higher in the elderly group than in the nonelderly group. Therefore, elderly patients with CAD may be treated by the transradial approach if their lesion can be treated without the strong backup support of a guiding system, a larger guiding system, or a double-access system. However, in the current study, the percentages of transfemoral approach and transradial approach were similar between the elderly and nonelderly patients. PCI in elderly patients tends to be difficult because of severe complex lesions (calcifications and ostial lesions, in particular). Therefore, there was no difference in the transfemoral approach rate in the 2 groups, despite the higher prevalence of comorbidities in the elderly group. In the near future, the development of less invasive PCI techniques that can be performed by the majority of cardiologists will result in improved PCI outcomes.

\section{Study Limitations}

Although this was a prospective, observational, multicenter cohort study, participating hospitals were not population-based, were not randomly selected, and were in Nagano Prefecture alone. Consecutive patients were enrolled in the current study; however, the decision to perform PCI was left to the discretion of the operator in all procedures, so selection bias could have occurred. Although the Euro Score is commonly used to predict short-term outcomes of cardiac surgery, there was insufficient data to calculate the Euro Score.

\section{Conclusions}

Although the in-hospital analysis of the SHINANO Registry revealed that the rates of procedural failure and in-hospital MACE were significantly higher in elderly patients than in nonelderly patients, the results were still feasible, particularly in patients who underwent elective PCI. Furthermore, our study revealed that elderly age was not a strong predictor of proce- dural failure and in-hospital ischemic events. Therefore, PCI should be performed in elderly patients without hesitation. In the decision to perform PCI, the presence of baseline comorbidities is a more important factor to consider than age alone.

\section{Disclosures}

Financial Support: None. Conflict of Interest: None of the authors have a real or perceived conflict of interest regarding the work in the manuscript. Author Contributions: All authors made substantial contributions to conception and design, acquisition of data, or analysis and interpretation of data, to drafting or revising the article, and approving the final version.

\section{References}

1. World Health Organization. Men, Ageing and health: Achieving health across the life span. Geneva: WHO, 2001.

2. Cabinet Office, Government of Japan. Annual report on the aging society: 2012.

3. Miura T, Soga Y, Doijiri T, Aihara H, Yokoi H, Iwabuchi M, et al. Prevalence and clinical outcome of polyvascular atherosclerotic disease in patients undergoing coronary intervention. Circ J 2013; 77: $89-95$.

4. TIME Investigators. Trial of invasive versus medical therapy in elderly patients with chronic symptomatic coronary-artery disease (TIME): A randomised trial. Lancet 2001; 358: 951-957.

5. Toyota T, Furukawa Y, Ehara N, Funakoshi S, Morimoto T, Kaji S, et al. Sex-based differences in clinical practice and outcomes for Japanese patients with acute myocardial infarction undergoing primary percutaneous coronary intervention. Circ J 2013; 77: 15081517.

6. Kaneko H, Yajima J, Oikawa Y, Tanaka S, Fukamachi D, Suzuki S, et al. Impact of aging on the clinical outcomes of Japanese patients with coronary artery disease after percutaneous coronary intervention. Heart Vessels 2014; 29: 156-164.

7. Batchelor WB, Anstrom KJ, Muhlbaier LH, Grosswald R, Weintranb WS, O'Neil WW, et al. Contemporary outcome trends in the elderly undergoing percutaneous coronary interventions: Results in 7,472 octogenarians. J Am Coll Cardiol 2000; 36: 723-730.

8. Yan BP, Gurvitch R, Duffy SJ, Clark DJ, Sebastian M, New G, et al. An evaluation of octogenarians undergoing percutaneous coronary intervention from the Melbourne Interventional Group registry. Catheter Cardiovasc Interv 2007; 70: 928-936.

9. From AM, Rihal CS, Lennon RJ, Holmes DR Jr, Prasad A. Temporal trends and improved outcomes of percutaneous coronary revascularization in nonagenarians. JACC Cardiovasc Interv 2008; 1: 692-698.

10. De Gregorio J, Kobayashi Y, Albiero R, Reimers B, Di Mario C, Finci L, et al. Coronary artery stenting in the elderly: Short-term outcome and long-term angiographic and clinical follow-up. J Am Coll Cardiol 1998; 32: 577-583.

11. Appleby CE, Ivanov J, Mackie K, Dzavik V, Overgaard CB. Inhospital outcomes of very elderly patients ( 85 years and older) undergoing percutaneous coronary intervention. Catheter Cardiovasc Interv 2011; 77: 634-641.

12. Klein LW, Block P, Brindis RG, McKay CR, McCallister BD, Wolk $\mathrm{M}$, et al. Percutaneous coronary interventions in octogenarians in the American College of Cardiology-National Cardiovascular Data Registry: Development of a nomogram predictive of in-hospital mortality. J Am Coll Cardiol 2002; 40: 394-402.

13. Varani E, Aquilina M, Balducelli M, Vecchi G, Frassineti V, Maresta A. Percutaneous coronary interventions in octogenarians: Acute and 12 month results in a large single-centre experience. Catheter Cardiovasc Interv 2009; 73: 449-454.

14. de Belder A, de la Torre Hernandez JM, Lopez-Palop R, O'Kane P, Hernandez F, Strange J, et al. A prospective randomized trial of everolimus-eluting stents versus bare metal stents in octogenarians: Xience or Vision stents for the management of angina in the elderly: The Xima Trial. J Am Coll Cardiol 2013 November 21, doi:10.1016/j. jacc.2013.10.053 [Epub ahead of print].

15. Feres F, Costa RA, Abizaid A, Leon MB, Marin-Neto JA, Botelho RV, et al. Three vs twelve months of dual antiplatelet therapy after zotarolimus-eluting stents: The OPTIMIZE randomized trial. JAMA 2013; 310: $2510-2522$.

16. Pancholy S, Patel T, Sanghvi K, Thomas M, Patel T. Comparison of door-to-balloon times for primary PCI using transradial versus transfemoral approach. Catheter Cadiovasc Interv 2010; 75: 991-995. 